\title{
EDITORIAL
}

\section{Differences between asthmatics and nonasthmatics hospitalised with influenza A infection}

\author{
Pierre Ernst*,\#, Amnon Ariel ${ }^{*}$ and Samy Suissa*,\#
}

$\mathbf{V}$ iral respiratory infections are the major cause of asthma exacerbations [1], while subjects with asthma are more likely to suffer significant morbidity when infected [2]. The severity and duration of respiratory insult depend on complex viral-host interactions. Abnormalities of the innate and adaptive immune response have been demonstrated in humans and in animal models of asthma [3]. These phenomena may explain the increased vulnerability of asthmatics to viral respiratory infections.

Although less common than rhinovirus, which is responsible for $33-55 \%$ of asthma exacerbations in children and adults, seasonal influenza infection is a well-recognised cause of asthma exacerbations (3-23\%) [1]. DAWOOD et al. [4] reported on a systematic surveillance programme for influenza-related hospitalisations of children in the USA. The proportion of children with asthma during seasonal influenza was 32\% from 2003 to 2009 , while $44 \%$ of children hospitalised with H1N1 in 2009 had asthma. O'RIORDAN et al. [5], in a paediatric hospitalbased series, also found asthma to be more frequent during the H1N1 influenza pandemic of 2009 than in previous years of seasonal influenza ( $22 \%$ versus $6 \%$ ). The remarkable increase in the risk of hospital admission in asthmatics with pandemic H1N1, compared with both seasonal influenza A and rhinovirus, may be explained by a lack of adaptive immunity to the H1N1 virus in many young asthmatic patients. Children with asthma were also more likely to require intensive care unit admission during the pandemic than with seasonal influenza A ( $22 \%$ versus $16 \%$ ) [4]. In a prospective study during the autumn of 2009, KLOEPFER et al. [6] collected nasal swabs for viral identification on a weekly basis. They found that children with asthma were more likely to become infected with H1N1 than children without asthma, while this was not the case for the other common respiratory viruses. Furthermore, H1N1 infection tended to be associated with more frequent loss of asthma control that rhinovirus. The increase in risk of an asthma exacerbation appears to be more pronounced with influenza A whether seasonal or pandemic, than with influenza B [4].

\footnotetext{
*Center for Clinical Epidemiology, Lady Davis Research Institute, Jewish General Hospital, Montreal, and ${ }^{\#}$ Depts of Epidemiology and Biostatistics, and Medicine, McGill University, Montreal, QC, Canada. "Lung Unit, Emek Medical Center, Afula, Israel.

CORRESPONDENCE: P. Ernst, Centre for Clinical Epidemiology, Jewish General Hospital, 3755 Cote Ste-Catherine, Montreal, Québec, Canada H3T 1E2. E-mail: pierre.ernst@mcgill.ca
}

While influenza A infection, including the H1N1 2009 pandemic, is associated with greater morbidity and mortality among patients with asthma as compared to the general population [7, 8], among those hospitalised for pandemic influenza, the presence of asthma has been associated with less severe outcomes [9]. This is in contradistinction to obesity, for example, which is associated with an important increase in severe outcomes, including death [9]. Among explanations proposed to explain the better prognosis of patients with asthma as compared to other patients hospitalised for influenza, are younger age and a lower threshold for admission with earlier presentation for inpatient care and a lesser degree of respiratory dysfunction.

In this issue of the journal, MYLES et al. [10] report on a cohort of 1520 individuals hospitalised in the UK with confirmed A/ H1N1 2009 infection. Clinical information was gathered prospectively by specially trained data collectors at the time of the hospital admission. Asthma was the most common comorbid illness (25.3\%). Patients with asthma were almost half as likely to have severe outcomes defined as the need for intensive support or death (OR $0.51,95 \%$ CI $0.36-0.72$ ). The lower morbidity among patients with asthma was not explained by milder respiratory dysfunction; rather subjects with asthma were more likely to have severe respiratory dysfunction at presentation. Adjustment for the difference in age distribution between patients with and without asthma did not modify the better prognosis associated with a diagnosis of asthma.

When accounting for the effect of pre-admission inhaled corticosteroids and admission to hospital within 4 days of the onset of symptoms, as well as several other covariates including the multiple triage criteria, the protective effect of asthma on severe outcomes is somewhat reduced and is no longer statistically significant (OR $0.62,95 \%$ CI $0.36-1.05$ ). The authors conclude that the use of inhaled corticosteroids and early admission to hospital explain most of the protective effect of asthma. It should be noted, however, that even if the protective effect of asthma becomes no longer statistically significant (the 95\% confidence interval includes 1) after adjustment for all covariates, including the use of inhaled corticosteroids and early admission to hospital, the odds ratio of 0.62 suggests that our best estimate is a $38 \%$ reduction in severe outcomes related to being labelled as having asthma.

The study by MYLES et al. [10] has several strengths, most significantly the prospective evaluation of a well-defined cohort with confirmed A/H1N1 2009 infection. This study is 
an excellent example of the efficient and powerful use of data mostly collected in the context of routine clinical practice. There are weaknesses, however: the diagnosis of asthma is not confirmed. Furthermore, we are unable to reliably distinguish between acute bronchospasm concurrent with the A/H1N1 2009 infection and exacerbation of prior asthma. Such new onset asthma or viral-induced bronchospasm is not infrequent with influenza, at least in children [11]. The prognosis of such cases in relation to influenza infection may be different. Furthermore, the apparent protective effect of inhaled corticosteroids and the reduced effect of asthma when adjusting for inhaled corticosteroid use may result, at least in part, from a validation of the diagnosis of asthma rather than a direct benefit of inhaled corticosteroids. Finally, the logistic regression approach to data analysis considers only the occurrence or non-occurrence of severe outcomes, but not the time to these outcomes. Even if the length of hospital stay does not vary much between asthmatics and nonasthmatics, rates per person-day and proportional hazard models can provide more accurate estimates of the effects of asthma on severe outcomes.

One no longer has to argue the benefits of inhaled corticosteroids in asthma. Their efficacy for symptom control and in preventing exacerbations is not disputed [12]. Their ability to prevent infectious exacerbations, usually viral, is less clear. One might think that reducing bronchial hyperresponsiveness and improving asthma control over time with inhaled corticosteroids should reduce the likelihood of a severe attack when a viral infection occurs. This is supported by the evidence that poor control and asthma severity are predictive of exacerbations $[2,12]$. However, viral exacerbations are more likely to be associated with neutrophilic inflammation which is refractory to corticosteroids. The interaction of atopy and viral infection may be particularly important in causing exacerbations [2, 12]. Influenza A infection has been associated with eosinophilic inflammation, especially in atopic individuals [13], possibly making it more likely to be attenuated by the regular use of inhaled corticosteroids.

The role of systemic corticosteroids in acute lung injury associated with viral infections including pandemic A/H1N1 2009 is controversial [14, 15]. The report of MYLES et al. [10] found that among patients with asthma, systemic corticosteroids were protective against severe outcomes (OR 0.36) while in patients without asthma they were associated with worse prognosis (OR 3.53). These estimates are unfortunately adjusted only for preadmission inhaled corticosteroids and admission to hospital within 4 days of the onset of symptoms, and may change with more complete adjustment for all covariates. Nevertheless, this suggests differences in the underlying pathophysiology of the respiratory dysfunction between the two groups. While rates of pneumonia were similar at admission, one suspects that, in patients with asthma, pulmonary infiltrates leading to a diagnosis of pneumonia may have been more often due to atelectasis and mucus plugging that are amenable to corticosteroid therapy.

In their prospective study of viral infections in children during the autumn of 2009, KLOEPFER et al. [6] estimate that $23 \%$ of exacerbations of asthma were due to H1N1. An effective vaccine for A/H1N1 2009 is available [16] providing an opportunity to prevent these exacerbations. The efficacy of seasonal influenza vaccine for prevention of asthma exacerbations is uncertain [2].
The greater importance of asthma as a comorbid condition in A/ H1N1 2009 suggests that an updated vaccination of all patients with persistent asthma may offer greater benefit during pandemic influenza.

In conclusion, patients with and without asthma contracting influenza H1N1 infection severe enough to require hospitalisation, probably differ as to the underling pathogenesis of the respiratory insult. Patients with asthma will tend to be in respiratory difficulty due to an exacerbation of their underlying asthma and, therefore, their prognosis may be positively influenced by prior use of inhaled corticosteroids and they may benefit from systemic corticosteroids while hospitalised. In contrast, patients without asthma in respiratory difficulty are probably suffering from uncontrolled pulmonary and systemic inflammation [17], unlikely to respond to corticosteroids and thus have a worse prognosis. The observed benefit of earlier referral to inpatient care, enabling earlier antiviral therapy, is a favourable prognostic factor in both asthmatic and nonasthmatic patients. Both groups are likely to benefit from vaccination.

\section{STATEMENT OF INTEREST}

Conflict of interest information can be found alongside the online version of this article at www.erj.ersjournals.com

\section{REFERENCES}

1 Xepapadaki P, Skevaki CL, Papadopoulos NG. Chapter 10. The role of viral and bacterial infections on the development and exacerbations of asthma. In: Carlsen $\mathrm{KH}$, Gerritsen J, eds. Paediatric Asthma. Eur Respir Monogr 2012; 56: 115-127.

2 Jackson DJ, Sykes A, Mallia P, et al. Asthma exacerbations: origin, effect, and prevention. J Allergy Clin Immunol 2011; 128: 1165-1174.

3 Holtzman MJ, Patel DA, Zhang Y, et al. Host epithelial-viral interactions as cause and cure for asthma. Curr Opin Immunol 2011; 23: 487-494.

4 Dawood FS, Kamimoto L, D'Mello TA, et al. Children with asthma hospitalized with seasonal or pandemic influenza, 2003-2009. Pediatrics 2011; 128: e27-e32.

5 O'Riordan S, Barton M, Yau Y, et al. Risk factors and outcomes among children admitted to hospital with pandemic H1N1 influenza. CMAJ 2010; 182: 39-44.

6 Kloepfer KM, Olenec JP, Lee WM, et al. Increased H1N1 infection rate in children with asthma. Am J Respir Crit Care Med 2012; 185: 1275-1279.

7 Miller EK, Griffin MR, Edwards KM, et al. Influenza burden for children with asthma. Pediatrics 2008; 121: 1-8.

8 Myles PR, Semple MG, Lim WS, et al. Predictors of clinical outcome in a national hospitalised cohort across both waves of the influenza A/H1N1 pandemic 2009-2010 in the UK. Thorax 2012; 67: 709-717.

9 Van Kerkhove MD, Vandemaele KA, Shinde V, et al. Risk factors for severe outcomes following 2009 influenza A (H1N1) infection: a global pooled analysis. PLoS Med 2011; 8: e1001053.

10 Myles P, Nguyen-Van-Tam JS, Semple MG, et al. Differences between asthmatics and non-asthmatics hospitalised with influenza A infection. Eur Respir J 2013; 41: 824-831.

11 Hasegawa S, Hirano R, Hashimoto $\mathrm{K}$, et al. Characteristics of atopic children with pandemic H1N1 influenza viral infection: pandemic H1N1 influenza reveals "occult" asthma of childhood. Pediatr Allergy Immunol 2011; 22: e119-e123.

12 O'Byrne PM. Therapeutic strategies to reduce asthma exacerbations. J Allergy Clin Immunol 2011; 128: 257-263.

13 Singh AM, Busse WW. Asthma exacerbations. 2: aetiology. Thorax 2006; 61: 809-816. 
14 Annane D. Pro: the illegitimate crusade against corticosteroids for severe H1N1 pneumonia. Am J Respir Crit Care Med 2011; 183: 1125-1126.

15 Matthay MA, Liu KD. Con: corticosteroids are not indicated for treatment of acute lung injury from H1N1 viral pneumonia. Am J Respir Crit Care Med 2011; 183: 1127-1128.
16 Busse WW, Peters SP, Fenton MJ, et al. Vaccination of patients with mild and severe asthma with a 2009 pandemic H1N1 influenza virus vaccine. J Allergy Clin Immunol 2011; 127: 130-137.

17 Bautista E, Chotpitayasunondh T, Gao Z, et al. Clinical aspects of pandemic 2009 influenza A (H1N1) virus infection. N Engl J Med 2010; 362: 1708-1719. 